\title{
Conditions for Strong Ellipticity of Anisotropic Elastic Materials
}

\author{
Deren Han · H.H. Dai · Liqun Qi
}

Received: 25 August 2007 / Published online: 20 May 2009

(C) Springer Science+Business Media B.V. 2009

\begin{abstract}
In this paper, we derive necessary and sufficient conditions for the strong ellipticity condition of anisotropic elastic materials. We first observe that the strong ellipticity condition holds if and only if a second order tensor function is positive definite for any unit vectors. Then we further link this condition to the rank-one positive definiteness of three second-order tensors, three fourth-order tensors and a sixth-order tensor. In particular, we consider conditions of strong ellipticity of the rhombic classes, for which we need to check the copositivity of three second-order tensors and the positive definiteness of a sixth-order tensor. A direct method is presented to verify our conditions.
\end{abstract}

Keywords Elasticity tensors · Anisotropic materials · Strong ellipticity $\cdot Z$-eigenvalues · Copositivity $\cdot$ Rhombic systems

Mathematics Subject Classification (2000) 74B20 $\cdot$ 74B10 15 A99

\section{Introduction}

The conditions of ordinary and strong ellipticity play important roles in nonlinear elasticity and materials. They are necessary or sufficient or both for an elastic material to have a

This work was supported by the Research Grant Council of Hong Kong.

D. Han

School of Mathematics and Computer Sciences, Nanjing Normal University, Nanjing, Jiangsu, China e-mail: handr00@hotmail.com

H.H. Dai

Department of Mathematics, The City University of Hong Kong, Kowloon Tong, Kowloon, Hong Kong e-mail: mahhdai@cityu.edu.hk

L. Qi (凶)

Department of Applied Mathematics, The Hong Kong Polytechnic University, Hung Hom, Kowloon, Hong Kong

e-mail: maqilq@polyu.edu.hk 
number of important statical and dynamical properties, e.g., in discussing uniqueness, wave propagation [6], instabilities due to the loss of ellipticity in nonlinear elasticity of fiberreinforced materials [8] and in the study of spatial behavior of the constrained anisotropic cylinders [2].

Most existing results on conditions for strong ellipticity focus on isotropic materials. It is well known [6] that in the particular case of an isotropic linearly elastic material, the strong ellipticity is equivalent to the fact that

$$
\mu>0, \quad \lambda+2 \mu>0
$$

where $\mu$ and $\lambda$ are the Lamé moduli. For planar problems, Knowles and Sternberg [7] characterized both strong and ordinary ellipticity for compressible isotropic nonlinearly materials, and Abeyaratne [1] proved similar results for ordinary ellipticity for incompressible materials. For three dimensional problems, Simpson and Spector [18] studied the strong ellipticity condition for isotropic nonlinearly elastic materials. Using the representation theorem for copositive matrices, they characterized necessary and sufficient conditions for compressible materials. Zee and Sternberg [21] characterized strong and ordinary ellipticity for incompressible isotropic materials. Alternative characterizations were proposed by Rosakis [17] and Wang and Aron [20] and a review on strong ellipticity for isotropic nonlinearly elastic materials was given by Dacorogna [5].

Characterizations of strong ellipticity like those cited above for isotropic materials have not yet been given for general anisotropic materials, and conditions for strong ellipticity for anisotropic elastic materials are very few and mainly focus on transversely isotropic linearly elastic solids. Notably, Payton [11], Padovani [10] and Merodio and Ogden [8] examined the strong ellipticity of such materials. In terms of the elastic constants $c_{11}, c_{33}, c_{55}, c_{12}$ and $c_{13}$ of the transversely isotropic linearly elastic solid, the strong ellipticity is equivalent to the following inequalities

$$
\begin{aligned}
c_{11} & >0, \quad c_{33}>0, \quad c_{55}>0, \quad c_{11}>c_{12}, \\
\left|c_{13}+c_{55}\right| & <c_{55}+\sqrt{c_{11} c_{33} .}
\end{aligned}
$$

Most recently, Chiriţă et al. [3] proposed some sufficient and necessary conditions for the strong ellipticity of anisotropic linearly elastic materials with certain symmetry properties (e.g., for the rhombic classes). For the general class of anisotropic elastic materials, Walton and Wilber [19] proposed some conditions for the strong ellipticity, which are only sufficient, but not necessary conditions.

In this paper, we establish the necessary and sufficient conditions for the strong ellipticity conditions of general anisotropic elastic materials through a completely different approach. More specifically, we connect the strong ellipticity of a tensor with the smallest $Z$ eigenvalues of some other tensors. The concept of $Z$-eigenvalues for tensors was introduced in [12] and further studied in [9, 13, 14]. A direct method for finding all the $Z$-eigenvalues of a tensor of dimension three was proposed in [16] in the third order case and extended to any order in [15]. We prove that a tensor has property of strong ellipticity if and only if the smallest $Z$-eigenvalues of some constitutive tensors are positive. For both planar and threedimensional cases, we have a direct method for finding all $Z$-eigenvalues of any tensor with even order and our conditions are easy to verify for general anisotropic elastic materials in this sense.

The paper is organized as follows. In the next section, we summarize some necessary concepts and preliminary results on the strong ellipticity and Z-eigenvalues. In Sect. 3, we 
propose and prove our conditions for strong ellipticity. In Sect. 4, we pay our attention to a class of special materials, i.e., the rhombic systems. For this special case, we propose sufficient and necessary conditions for strong ellipticity, which involve to check the copositivity of three second-order tensors and the positivity of the smallest $Z$-eigenvalue of a sixth-order tensor. Section 5 describes the direct method for finding all the $Z$-eigenvalues of a tensor of dimension three and any even order. This makes our conditions easy to check. Section 6 consists of some final conclusions.

\section{Ellipticity and Rank-One Positive Definiteness}

A fourth-order $n$-dimensional tensor $\mathcal{A}$ consists of $n^{4}$ real entries

$$
a_{i j k l} \in \Re, \quad i, j, k, l=1, \ldots, n .
$$

For elastic materials, the components $a_{i j k l}$ of the tensor of elastic moduli satisfy the symmetries

$$
a_{i j k l}=a_{k j i l}=a_{i l k j} .
$$

The strong ellipticity condition states that

$$
f(x, y) \equiv \mathcal{A} x y x y \equiv \sum_{i, j, k, l=1}^{n} a_{i j k l} x_{i} y_{j} x_{k} y_{l}>0,
$$

for all nonzero vectors $x, y \in \mathfrak{R}^{n}$. Here, we use the notation in [12-14] for tensors and vectors and $x=\left(x_{1}, \ldots, x_{n}\right)^{\top}$ and $y=\left(y_{1}, \ldots, y_{n}\right)^{\top}$ are two vectors in $\Re^{n}$. If the above inequality holds with equality, i.e.,

$$
\sum_{i, j, k, l=1}^{n} a_{i j k l} x_{i} y_{j} x_{k} y_{l} \geq 0,
$$

for all $x, y \in \Re^{n}$, then we say that the tensor $\mathcal{A}$ satisfy the ordinary ellipticity. Strong and ordinary ellipticity conditions are of importance in order for an elastic material to have a number of important statical and dynamical properties.

It is easy to see that the strong ellipticity condition (2) holds if and only if the optimal value of the following global polynomial optimization problem is positive

$$
\begin{array}{ll}
\min & f(x, y) \equiv \mathcal{A} x y x y \equiv \sum_{i, j, k, l=1}^{n} a_{i j k l} x_{i} y_{j} x_{k} y_{l} \\
\text { s.t. } & x^{\top} x=1, \\
& y^{\top} y=1,
\end{array}
$$

where $x, y \in \Re^{n}$; and the ordinary ellipticity conditions holds if and only if the optimal value of (3) is nonnegative.

Denote $\mathcal{A} \cdot y x y$ as a vector whose $i$ th component is $\sum_{j, k, l=1}^{n} a_{i j k l} y_{j} x_{k} y_{l}$, and $\mathcal{A} x y x \cdot$ as a vector whose $l$ th component is $\sum_{i, j, k=1}^{n} a_{i j k l} x_{i} y_{j} x_{k}$. The optimality condition of (3) is:

$$
\left\{\begin{array}{l}
\mathcal{A} \cdot y x y=\lambda x, \\
\mathcal{A} x y x \cdot=\mu y, \\
x^{\top} x=1, \\
y^{\top} y=1 .
\end{array}\right.
$$


Suppose $\lambda, \mu, x$ and $y$ satisfy (4). It is easy to see that

$$
\lambda=\mathcal{A x y x y}=\mu \text {. }
$$

Thus, we may rewrite (4) as

$$
\left\{\begin{array}{l}
\mathcal{A} \cdot y x y=\lambda x, \\
\mathcal{A} x y x=\lambda y, \\
x^{\top} x=1, \\
y^{\top} y=1 .
\end{array}\right.
$$

If $\lambda \in \Re, x \in \Re^{n}$ and $y \in \Re^{n}$ satisfy (5), we call $\lambda$ an $M$-eigenvalue of $\mathcal{A}$, and call $x$ and $y$ left and right $M$-eigenvectors of $\mathcal{A}$, associated with the $M$-eigenvalue $\lambda$. Here, the letter " $M$ " stands for mechanics.

It is easy to see that $M$-eigenvalues always exist and the strong ellipticity condition holds if and only if the smallest $M$-eigenvalue of $\mathcal{A}$ is positive. For $n=2$, we may construct a direct method to find all the $M$-eigenvalues of $\mathcal{A}$, but for $n=3$, it is difficult to do this.

The elasticity tensor $\mathcal{A}$ is called rank-one positive definite if for all $x \in \Re^{n}, x \neq 0$,

$$
f(x, x) \equiv \mathcal{A} x^{4} \equiv \mathcal{A} x x x x \equiv \sum_{i, j, k, l=1}^{n} a_{i j k l} x_{i} x_{j} x_{k} x_{l}>0 .
$$

Clearly, if the strong ellipticity holds, then $\mathcal{A}$ is rank-one positive definite and the reverse conclusion is not true.

It is also easy to see that the rank-one positivity condition (6) holds if and only if the optimal value of the following global polynomial optimization problem is positive

$$
\begin{array}{ll}
\min & f(x, x) \equiv \mathcal{A} x^{4} \equiv \sum_{i, j, k, l=1}^{n} a_{i j k l} x_{i} x_{j} x_{k} x_{l} \\
\text { s.t. } & x^{\top} x=1 .
\end{array}
$$

The optimality condition for (7) is:

$$
\left\{\begin{array}{l}
\mathcal{A} x^{3}=\lambda x \\
x^{\top} x=1
\end{array}\right.
$$

where $\mathcal{A} x^{3}=\mathcal{A} \cdot x x x=\mathcal{A} x x x \cdot$. In [12-14], it was defined that if $\lambda \in \Re$ and $x \in \Re^{n}$ satisfy (8), $\lambda$ is called a $Z$-eigenvalue of $\mathcal{A}$, and $x$ is called the $Z$-eigenvector of $\mathcal{A}$, associated with the $Z$-eigenvalue $\lambda$. Thus, if the smallest $Z$-eigenvalue of $\mathcal{A}$ is positive, then $\mathcal{A}$ is rank-one positive. Not like the $M$-eigenvalue, for both $n=2$ and $n=3$, we may construct direct methods to find all $Z$-eigenvalues and the associated $Z$-eigenvectors for an elasticity tensor $\mathcal{A}$.

Another tool we will use to establish our conditions for the strong ellipticity of an elastic tensor $\mathcal{A}$ is the strict copositivity of a second-order tensor. A second-order tensor is represented by an $n \times n$ matrix in a coordinate system. An $n \times n$ matrix $M$ is said to be strictly copositive if

$$
v^{\top} M v>0, \text { whenever } \quad v_{i} \geq 0 \quad \text { and } \quad v \neq 0 .
$$

For three-dimensional matrices, Simpson and Spector [18] obtained the following equivalent conditions for strict copositivity. 
Lemma 1 Let $M=\left(m_{i j}\right), i, j=1,2,3$ be a symmetric matrix. Then, $M$ is strictly copositive if and only if the following conditions hold:

$$
\begin{aligned}
& m_{11}>0, \quad m_{22}>0, \quad m_{33}>0, \\
& R \equiv m_{12}+\left(m_{11} m_{22}\right)^{\frac{1}{2}}>0, \quad S \equiv m_{13}+\left(m_{11} m_{33}\right)^{\frac{1}{2}}>0, \\
& T \equiv m_{23}+\left(m_{22} m_{33}\right)^{\frac{1}{2}}>0, \\
& m_{33}^{\frac{1}{2}} m_{12}+m_{22}^{\frac{1}{2}} m_{13}+m_{11}^{\frac{1}{2}} m_{23}+(2 R S T)^{\frac{1}{2}}+\left(m_{11} m_{22} m_{33}\right)^{\frac{1}{2}}>0 .
\end{aligned}
$$

\section{Conditions for Ellipticity}

For $n=2$, it is possible to construct a direct method for finding all the $M$-eigenvalues of $\mathcal{A}$. This forms checkable conditions for the strong ellipticity of an elastic tensor $\mathcal{A}$ when $n=2$. We now pay our attention to the case that $n=3$.

Let $Q(x)$ denote the acoustic tensor, with components defined by

$$
q_{j l}=\sum_{i, k=1}^{3} a_{i j k l} x_{i} x_{k} .
$$

Then the strong ellipticity of $\mathcal{A}$ is equivalent to the positive definiteness of $Q(x)$ for all unit vector $x$.

Since $Q(x)$ is a symmetric matrix for any $x \in \mathfrak{R}^{3}, Q(x)$ is positive definite if and only if all its minors are positive definite. We first consider its diagonal entries, i.e., $q_{11}, q_{22}, q_{33}$.

From (10) we have

$$
q_{11}=a_{1111} x_{1}^{2}+2 a_{1121} x_{1} x_{2}+2 a_{1131} x_{1} x_{3}+a_{2121} x_{2}^{2}+2 a_{2131} x_{2} x_{3}+a_{3131} x_{3}^{2} .
$$

Thus, we have

$$
q_{11}>0 \quad \text { for all } x \neq 0 \quad \Longleftrightarrow \quad M_{1} \text { is positively definite, }
$$

where $M_{1}$ is the matrix defined by

$$
M_{1} \equiv\left(\begin{array}{lll}
a_{1111} & a_{1121} & a_{1131} \\
a_{1121} & a_{2121} & a_{2131} \\
a_{1131} & a_{2131} & a_{3131}
\end{array}\right) .
$$

In a similar way, we have that

$$
q_{22}=a_{1212} x_{1}^{2}+2 a_{1222} x_{1} x_{2}+2 a_{1232} x_{1} x_{3}+a_{2222} x_{2}^{2}+2 a_{2232} x_{2} x_{3}+a_{3232} x_{3}^{2}
$$

and

$$
q_{22}>0 \quad \text { for all } x \neq 0 \quad \Longleftrightarrow \quad M_{2} \text { is positively definite, }
$$

where $M_{2}$ is the matrix defined by

$$
M_{2} \equiv\left(\begin{array}{lll}
a_{1212} & a_{1222} & a_{1232} \\
a_{1222} & a_{2222} & a_{2232} \\
a_{1232} & a_{2232} & a_{3232}
\end{array}\right) .
$$


We also have that

$$
q_{33}=a_{1313} x_{1}^{2}+2 a_{1323} x_{1} x_{2}+2 a_{1333} x_{1} x_{3}+a_{2323} x_{2}^{2}+2 a_{2333} x_{2} x_{3}+a_{3333} x_{3}^{2}
$$

and

$$
q_{22}>0 \quad \text { for all } x \neq 0 \quad \Longleftrightarrow \quad M_{3} \text { is positively definite, }
$$

where $M_{2}$ is the matrix defined by

$$
M_{3} \equiv\left(\begin{array}{lll}
a_{1313} & a_{1323} & a_{1333} \\
a_{1323} & a_{2323} & a_{2333} \\
a_{1333} & a_{2333} & a_{3333}
\end{array}\right) .
$$

Now, we consider the second-order minors. That is

$$
q_{11} q_{22}-q_{12}^{2}>0, \quad q_{11} q_{33}-q_{13}^{2}>0, \quad q_{22} q_{33}-q_{23}^{2}>0, \quad \text { for all } x \neq 0,
$$

where

$$
\begin{aligned}
& q_{12}=a_{1112} x_{1}^{2}+2 a_{1122} x_{1} x_{2}+2 a_{1132} x_{1} x_{3}+a_{2122} x_{2}^{2}+2 a_{2132} x_{2} x_{3}+a_{3132} x_{3}^{2}, \\
& q_{13}=a_{1113} x_{1}^{2}+2 a_{1123} x_{1} x_{2}+2 a_{1133} x_{1} x_{3}+a_{2123} x_{2}^{2}+2 a_{2133} x_{2} x_{3}+a_{3133} x_{3}^{2},
\end{aligned}
$$

and

$$
q_{23}=a_{1213} x_{1}^{2}+2 a_{1223} x_{1} x_{2}+2 a_{1233} x_{1} x_{3}+a_{2223} x_{2}^{2}+2 a_{2233} x_{2} x_{3}+a_{3233} x_{3}^{2} .
$$

Let $\mathcal{T}^{1}, \mathcal{T}^{2}$ and $\mathcal{T}^{3}$ be the fourth-order three-dimensional tensors, such that

$$
\begin{aligned}
& \mathcal{T}^{1} x^{4} \equiv \sum_{i, j, k, l=1}^{3} t_{i j k l}^{1} x_{i} x_{j} x_{k} x_{l}=q_{11} q_{22}-q_{12}^{2}, \\
& \mathcal{T}^{2} x^{4} \equiv \sum_{i, j, k, l=1}^{3} t_{i j k l}^{2} x_{i} x_{j} x_{k} x_{l}=q_{11} q_{33}-q_{13}^{2},
\end{aligned}
$$

and

$$
\mathcal{T}^{3} x^{4} \equiv \sum_{i, j, k, l=1}^{3} t_{i j k l}^{3} x_{i} x_{j} x_{k} x_{l}=q_{22} q_{33}-q_{23}^{2} .
$$

Then, (17) is equivalent to the positivity of the smallest $Z$-eigenvalues of the tensors $\mathcal{T}^{1}, \mathcal{T}^{2}$ and $\mathcal{T}^{3}$.

Finally, it requires that

$$
\operatorname{det} Q(x)>0, \text { for all } x \neq 0 \text {. }
$$

Note that

$$
\operatorname{det} Q(x)=q_{11}\left(q_{22} q_{33}-q_{23}^{2}\right)-q_{12}\left(q_{12} q_{33}-q_{13} q_{23}\right)+q_{13}\left(q_{12} q_{23}-q_{13} q_{22}\right),
$$


and since each $q_{i j}$ is a quadratic form, $i, j=1,2,3$, det $Q(x)$ is a homogeneous polynomial with degree 6 . Let $\mathcal{W}$ be the sixth-order three-dimensional tensor such that

$$
\mathcal{W} x^{6} \equiv \sum_{i_{1}, \ldots, i_{6}=1}^{3} w_{i_{1} \cdots i_{6}} x_{i_{1}} x_{i_{2}} \cdots x_{i_{6}}=\operatorname{det} Q(x)
$$

Then (18) holds if and only if the smallest $Z$-eigenvalue of $\mathcal{W}$ is positive.

Summarizing the above results, we have the following theorem.

Theorem 1 An elastic tensor $\mathcal{A}$ is strongly elliptic if and only if the following conditions hold

1. The matrices $M_{1}, M_{2}$ and $M_{3}$ are positively definite;

2. The fourth-order tensors $\mathcal{T}^{1}, \mathcal{T}^{2}$ and $\mathcal{T}^{3}$ are rank-one positive;

3. The sixth-order tensor $\mathcal{W}$ is rank-one positive.

\section{Rhombic Systems}

In this section, we discuss the strong ellipticity for the rhombic system, where the elasticity tensor $\mathcal{A}$ has the following property:

$$
\begin{aligned}
& a_{1123}=a_{1131}=a_{1112}=a_{2223}=a_{2231}=a_{2212}=0, \\
& a_{3323}=a_{3331}=a_{3312}=a_{2331}=a_{2312}=a_{3112}=0 .
\end{aligned}
$$

As in [3], we denote the only nonzero components of the elasticity tensor $\mathcal{A}$ as follows

$$
\begin{aligned}
& a_{11}=a_{1111}, \quad a_{22}=a_{2222}, \quad a_{33}=a_{3333}, \quad a_{12}=a_{1122}, \quad a_{23}=a_{2233}, \\
& a_{31}=a_{3311}, \quad a_{44}=a_{2323}, \quad a_{55}=a_{1313}, \quad a_{66}=a_{1212} .
\end{aligned}
$$

For this special case, we have the following result.

Theorem 2 Let $\mathcal{A}$ be an elastic tensor whose components satisfy conditions (21)-(22). Then $\mathcal{A}$ is strongly elliptic if and only if the following conditions hold

1. $a_{11}>0, a_{22}>0, a_{33}>0, a_{44}>0, a_{55}>0, a_{66}>0$;

2. The matrices $P^{1}, P^{2}$ and $P^{3}$ defined in (24)-(28) are copositive;

3. The sixth-order tensor $\mathcal{W}$ is rank-one positive.

Proof It follows from Theorem 1 that $\mathcal{A}$ has the strong ellipticity property if and only if Items $1-3$ hold. Since the elements of $\mathcal{A}$ satisfy conditions (21)-(22), we have

$$
\begin{aligned}
M_{1} & =\left(\begin{array}{ccc}
a_{11} & 0 & 0 \\
0 & a_{66} & 0 \\
0 & 0 & a_{55}
\end{array}\right), \\
M_{2} & =\left(\begin{array}{ccc}
a_{66} & 0 & 0 \\
0 & a_{22} & 0 \\
0 & 0 & a_{44}
\end{array}\right),
\end{aligned}
$$


and

$$
M_{3}=\left(\begin{array}{ccc}
a_{55} & 0 & 0 \\
0 & a_{44} & 0 \\
0 & 0 & a_{33}
\end{array}\right) .
$$

Thus, $M_{1}, M_{2}$ and $M_{3}$ are positive definite if and only if their diagonal elements are positive. This proves Item 1.

From (21)-(22), we have

$$
\begin{aligned}
& q_{11}=a_{11} x_{1}^{2}+a_{66} x_{2}^{2}+a_{55} x_{3}^{2}, \\
& q_{22}=a_{66} x_{1}^{2}+a_{22} x_{2}^{2}+a_{44} x_{3}^{2}, \\
& q_{33}=a_{55} x_{1}^{2}+a_{44} x_{2}^{2}+a_{33} x_{3}^{2}, \\
& q_{12}=2 a_{12} x_{1} x_{2}, \\
& q_{13}=2 a_{31} x_{1} x_{3},
\end{aligned}
$$

and

$$
q_{23}=2 a_{23} x_{2} x_{3}
$$

Thus,

$$
\begin{aligned}
q_{11} q_{22} & -q_{12}^{2} \\
= & a_{11} a_{66} x_{1}^{4}+\left(a_{11} a_{22}+a_{66}^{2}-4 a_{12}^{2}\right) x_{1}^{2} x_{2}^{2}+\left(a_{11} a_{44}+a_{55} a_{66}\right) x_{1}^{2} x_{3}^{2} \\
& +a_{22} a_{66} x_{2}^{4}+\left(a_{44} a_{66}+a_{22} a_{55}\right) x_{2}^{2} x_{3}^{2}+a_{44} a_{55} x_{3}^{4} \\
= & \left(\begin{array}{l}
x_{1}^{2} \\
x_{2}^{2} \\
x_{3}^{2}
\end{array}\right)^{\top} P^{1}\left(\begin{array}{l}
x_{1}^{2} \\
x_{2}^{2} \\
x_{3}^{2}
\end{array}\right)
\end{aligned}
$$

where

$$
P^{1} \equiv\left(\begin{array}{ccc}
a_{11} a_{66} & \frac{\left(a_{11} a_{22}+a_{66}^{2}-4 a_{12}^{2}\right)}{2} & \frac{\left(a_{11} a_{44}+a_{55} a_{66}\right)}{2} \\
\frac{\left(a_{11} a_{22}+a_{66}^{2}-4 a_{12}^{2}\right)}{2} & a_{22} a_{66} & \frac{\left(a_{44} a_{66}+a_{22} a_{55}\right)}{2} \\
\frac{\left(a_{11} a_{44}+a_{55} a_{66}\right)}{2} & \frac{\left(a_{44} a_{66}+a_{22} a_{55}\right)}{2} & a_{44} a_{55}
\end{array}\right) .
$$

We then have

$$
q_{11} q_{22}-q_{12}^{2}>0 \quad \text { for all } x \neq 0 \quad \Longleftrightarrow \quad P^{1} \text { is copositive. }
$$

Similarly,

$$
\begin{aligned}
q_{11} q_{33} & -q_{13}^{2} \\
= & a_{11} a_{55} x_{1}^{4}+\left(a_{11} a_{44}+a_{55} a_{66}\right) x_{1}^{2} x_{2}^{2}+\left(a_{11} a_{33}+a_{55}^{2}-4 a_{31}^{2}\right) x_{1}^{2} x_{3}^{2} \\
& +a_{44} a_{66} x_{2}^{4}+\left(a_{33} a_{66}+a_{44} a_{55}\right) x_{2}^{2} x_{3}^{2}+a_{33} a_{55} x_{3}^{4} \\
= & \left(\begin{array}{l}
x_{1}^{2} \\
x_{2}^{2} \\
x_{3}^{2}
\end{array}\right) P^{\top}\left(\begin{array}{l}
x_{1}^{2} \\
x_{2}^{2} \\
x_{3}^{2}
\end{array}\right)
\end{aligned}
$$


where

$$
P^{2} \equiv\left(\begin{array}{ccc}
a_{11} a_{55} & \frac{\left(a_{11} a_{44}+a_{55} a_{66}\right)}{2} & \frac{\left(a_{11} a_{33}+a_{55}^{2}-4 a_{31}^{2}\right)}{2} \\
\frac{\left(a_{11} a_{44}+a_{55} a_{66}\right)}{2} & a_{44} a_{66} & \frac{\left(a_{33} a_{66}+a_{44} a_{55}\right)}{2} \\
\frac{\left(a_{11} a_{33}+a_{55}^{2}-4 a_{31}^{2}\right)}{2} & \frac{\left(a_{33} a_{66}+a_{44} a_{55}\right)}{2} & a_{33} a_{55}
\end{array}\right)
$$

and

$$
q_{11} q_{33}-q_{13}^{2}>0 \quad \text { for all } x \neq 0 \quad \Longleftrightarrow \quad P^{2} \text { is copositive; }
$$

and

$$
\begin{aligned}
q_{22} q_{33} & -q_{23}^{2} \\
= & a_{55} a_{66} x_{1}^{4}+\left(a_{44} a_{66}+a_{22} a_{55}\right) x_{1}^{2} x_{2}^{2}+\left(a_{33} a_{66}+a_{44} a_{55}\right) x_{1}^{2} x_{3}^{2} \\
& +a_{22} a_{44} x_{2}^{4}+\left(a_{22} a_{33}+a_{44}^{2}-4 a_{23}^{2}\right) x_{2}^{2} x_{3}^{2}+a_{33} a_{44} x_{3}^{4} \\
= & \left(\begin{array}{l}
x_{1}^{2} \\
x_{2}^{2} \\
x_{3}^{2}
\end{array}\right) P^{\top}\left(\begin{array}{l}
x_{1}^{2} \\
x_{2}^{2} \\
x_{3}^{2}
\end{array}\right)
\end{aligned}
$$

where

$$
P^{3} \equiv\left(\begin{array}{ccc}
a_{55} a_{66} & \frac{\left(a_{44} a_{66}+a_{22} a_{55}\right)}{2} & \frac{\left(a_{33} a_{66}+a_{44} a_{55}\right)}{2} \\
\frac{\left(a_{44} a_{66}+a_{22} a_{55}\right)}{2} & a_{22} a_{44} & \frac{\left(a_{22} a_{33}+a_{44}^{2}-4 a_{23}^{2}\right)}{2} \\
\frac{\left(a_{33} a_{66}+a_{44} a_{55}\right)}{2} & \frac{\left(a_{22} a_{33}+a_{44}^{2}-4 a_{23}^{2}\right)}{2} & a_{33} a_{44}
\end{array}\right)
$$

and

$$
q_{22} q_{33}-q_{23}^{2}>0 \quad \text { for all } x \neq 0 \quad \Longleftrightarrow \quad P^{3} \text { is copositive. }
$$

\section{A Direct Method for the Smallest $Z$-Eigenvalue and Its $Z$-Eigenvectors}

The main task to verify the equivalent conditions for strong ellipticity of an elasticity tensor $\mathcal{A}$ is to find the smallest $Z$-eigenvalues of three fourth-order dimensional tensors and one sixth-order three dimensional tensor for the general case; or merely the smallest $Z$ eigenvalues of a sixth-order three dimensional tensor, for the special case of rhombic systems. In this section, we provide a direct method for finding all the $Z$-eigenvalues of an $m$ th-order three-dimensional tensor. That is, we solve the following optimization problem

$$
\begin{array}{ll}
\min & f(x) \equiv \mathcal{A} x^{m} \equiv \sum_{i_{1}, \ldots, i_{m}=1}^{3} a_{i_{1} \cdots i_{m}} x_{i_{1}} \cdots x_{i_{m}} \\
\text { s.t. } & x^{\top} x=1
\end{array}
$$

by finding all $\lambda \in \Re$, such that the optimality condition for (29) holds, i.e.,

$$
\left\{\begin{array}{l}
\mathcal{A} x^{m-1}=\lambda x, \\
x^{\top} x=1,
\end{array}\right.
$$


where $\mathcal{A} x^{m-1}$ is a vector in $\Re^{n}$ whose $i$ th component is

$$
\left(\mathcal{A} x^{m-1}\right)_{i}=\sum_{i_{2}, \ldots, i_{m}=1}^{3} a_{i i_{2} \cdots i_{m}} x_{i_{2}} \cdots x_{i_{m}} .
$$

This method was proposed by Qi, Wang and Wang in [16] in the case $m=3$ and extended to arbitrary $m$ in [15]. We describe this method here for completeness.

The key idea here is to reduce the four variable system (30) to a system involving only two variables. Then, for this system of two variables, we may use the Sylvester formula of the resultant to find the solutions.

Let

$$
\alpha_{j}=a_{i_{1} \cdots i_{m}}
$$

for $i_{1}=\cdots=i_{m-j}=1, i_{m-j+1}=\cdots=i_{m}=2$ and $0 \leq j \leq m-1$. Furthermore, for $0 \leq i$, $j \leq m-1$, denote

$$
\left(\begin{array}{c}
m-1 \\
i, j
\end{array}\right)=\frac{(m-1) !}{i ! j !(m-1-i-j) !}
$$

$\beta_{j}=a_{3 i_{1} \cdots i_{m}-1}$, for $i_{1}=\cdots=i_{m-1-j}=1, i_{m-j}=\cdots=i_{m-1}=2$, and $\gamma_{k, i, j}=$ $a_{k} \underbrace{1 \cdots 1}_{i} \underbrace{2 \cdots 2}_{j} \underbrace{3 \cdots 3}_{m-1-i-j}$ for $k=1,2,3$.

We have the following theorem.

Theorem 3 (Qi, Wang and Wang) Let $\mathcal{A}$ be an mth-order three-dimensional tensor, where $m$ is even. We have the following results on the Z-eigenvalues and their corresponding $Z$ eigenvectors.

(a) If $a_{11 \cdots 12}=a_{11 \cdots 13}=0$, then $\lambda=a_{11 \cdots 1}$ is a Z-eigenvalue of $\mathcal{A}$ and the corresponding $Z$-eigenvector is $x=(1,0,0)^{\top}$.

(b) For any real roots $t$ of the following equations:

$$
\begin{aligned}
& \left\{\begin{array}{l}
\sum_{j=0}^{m-1}\left(\begin{array}{c}
m-1 \\
j
\end{array}\right)\left[\alpha_{j} t^{m-j-1}-\alpha_{j+1} t^{m-j}\right]=0, \\
\sum_{j=0}^{m-1}\left(\begin{array}{c}
m-1 \\
j
\end{array}\right) \beta_{j} t^{m-j-1}=0,
\end{array}\right. \\
& x= \pm \frac{1}{\sqrt{t^{2}+1}}(t, 1,0)^{\top}
\end{aligned}
$$

is a Z-eigenvector corresponding to the Z-eigenvalue $\lambda=\mathcal{A} x^{m}$.

(c) For any real roots $(u, v)^{\top}$ of the following equations:

$$
\left\{\begin{array}{l}
b_{m-1}^{3}(v) u^{m}+\sum_{i=1}^{m-1}\left[b_{i-1}^{3}(v)-b_{i}^{1}(v)\right] u^{i}-b_{0}^{1}(v)=0 \\
\sum_{i=1}^{m-1}\left[b_{i}^{3}(v) v-b_{i}^{2}(v)\right] u^{i}=0
\end{array}\right.
$$


where

$$
b_{i}^{k}(v)=\sum_{j=0}^{m-1-i}\left(\begin{array}{c}
m-1 \\
i, j
\end{array}\right) \gamma_{k, i, j} v^{j}, \quad \begin{aligned}
& k=1,2,3, \\
& i=0,1, \ldots, m-1
\end{aligned}
$$

$\lambda=\mathcal{A} x^{m}$ is a Z-eigenvalue with the corresponding eigenvector

$$
x= \pm \frac{1}{\sqrt{u^{2}+v^{2}+1}}(u, v, 1)^{\top} .
$$

All the Z-eigenvalues and the associated Z-eigenvectors are given by (a), (b) and (c) if $a_{11 \cdots 12}=a_{11 \cdots 13}=0$, and by (b) and (c) otherwise.

Proof It is easy to check that if $a_{11 \cdots 12}=a_{11 \cdots 13}=0$, then $\lambda=a_{11 \cdots 1}$ and $x=(1,0,0)^{\top}$ satisfy (30); hence $\lambda=a_{11 \cdots 1}$ is a $Z$-eigenvalue of $\mathcal{A}$ with the corresponding $Z$-eigenvector $x=(1,0,0)^{\top}$. This proves the conclusion (a).

If $x_{2} \neq 0$ and $x_{3}=0$, then (30) becomes

$$
\left\{\begin{array}{l}
\sum_{j=0}^{m-1}\left(\begin{array}{c}
m-1 \\
j
\end{array}\right) \alpha_{j} x_{1}^{m-1-j} x_{2}^{j}=\lambda x_{1} \\
\sum_{j=0}^{m-1}\left(\begin{array}{c}
m-1 \\
j
\end{array}\right) \alpha_{j+1} x_{1}^{m-1-j} x_{2}^{j}=\lambda x_{2} \\
\sum_{j=0}^{m-1}\left(\begin{array}{c}
m-1 \\
j
\end{array}\right) \beta_{j} x_{1}^{m-1-j} x_{2}^{j}=0 \\
x_{1}^{2}+x_{2}^{2}=1
\end{array}\right.
$$

Let $t=x_{1} / x_{2}$. From the first three equations of (35), we have (31). By the fourth equation of (35), we have (32). This proves (b).

If $x_{3} \neq 0$, then (30) becomes

$$
\left\{\begin{array}{l}
\sum_{i=0}^{m-1} \sum_{j=0}^{m-1-i}\left(\begin{array}{c}
m-1 \\
i, j
\end{array}\right) \gamma_{1, i, j} x_{1}^{i} x_{2}^{j} x_{3}^{m-1-i-j}=\lambda x_{1}, \\
\sum_{i=0}^{m-1} \sum_{j=0}^{m-1-i}\left(\begin{array}{c}
m-1 \\
i, j
\end{array}\right) \gamma_{2, i, j} x_{1}^{i} x_{2}^{j} x_{3}^{m-1-i-j}=\lambda x_{2}, \\
\sum_{i=0}^{m-1} \sum_{j=0}^{m-1-i}\left(\begin{array}{c}
m-1 \\
i, j
\end{array}\right) \gamma_{3, i, j} x_{1}^{i} x_{2}^{j} x_{3}^{m-1-i-j}=\lambda x_{3}, \\
x_{1}^{2}+x_{2}^{2}+x_{3}^{2}=1 .
\end{array}\right.
$$

Let $u=x_{1} / x_{3}$ and $v=x_{2} / x_{3}$. From the first three equations of (36), we obtain

$$
\left\{\begin{array}{l}
\sum_{i=0}^{m-1} \sum_{j=0}^{m-1-i}\left(\begin{array}{c}
m-1 \\
i, j
\end{array}\right)\left(\gamma_{1, i, j} u^{i} v^{j}-\gamma_{3, i, j} u^{i+1} v^{j}\right)=0 \\
\sum_{i=0}^{m-1} \sum_{j=0}^{m-1-i}\left(\begin{array}{c}
m-1 \\
i, j
\end{array}\right)\left(\gamma_{2, i, j} u^{i} v^{j}-\gamma_{3, i, j} u^{i} v^{j+1}\right)=0 .
\end{array}\right.
$$


That is,

$$
\left\{\begin{array}{l}
-\gamma_{3, m-1,0} u^{m}+\sum_{i=1}^{m-1}\left(\sum_{j=0}^{m-1-i}\left(\begin{array}{c}
m-1 \\
i, j
\end{array}\right) \gamma_{1, i, j}-\sum_{j=0}^{m-i}\left(\begin{array}{c}
m-1 \\
i-1, j
\end{array}\right) \gamma_{3, i-1, j}\right) u^{i} v^{j} \\
+\sum_{j=0}^{m-1}\left(\begin{array}{c}
m-1 \\
j
\end{array}\right) \gamma_{1,0, j} v^{j}=0 \\
\sum_{i=0}^{m-1}\left(\begin{array}{c}
m-1-i \\
\sum_{j=0}^{m-1}\left(\begin{array}{c}
m-1 \\
i, j
\end{array}\right) \gamma_{2, i, j} v^{j}-\gamma_{3, i, j} v^{j+1}
\end{array}\right) u^{i}=0
\end{array}\right.
$$

which is just (33). By the definition of (36), we have (34). This completes the conclusion of Item (c) and the whole theorem.

From the above theorem, we can see that to find all the $Z$-eigenvalues and the associated $Z$-eigenvectors, we need to solve some systems of polynomial equations with two variables. To solve such systems, we can use the resultant method from algebraic geometry [4]. For example, to solve (33), we may regard it as equations for $u$ with coefficients $b_{k}^{i}(v)$, functions in $v$. The system (33) has solutions if and only if its resultant vanishes [4]. By the Sylvester theorem [4], its resultant can be calculated as a determinant of an $m \times m$ matrix, which is a polynomial of $v$. We can use Matlab to find all of its real roots. After this, we substitute them to (33) to find all the real solutions of $u$. Correspondingly, all the $Z$-eigenvalues and the associated $Z$-eigenvectors can be found.

\section{Final Remarks}

We establish some necessary and sufficient conditions for strong ellipticity of general anisotropic elastic materials, which need to check the positive definiteness of three secondorder tensors and rank-one positive definiteness of three fourth-order and a sixth-order threedimensional tensor. We then pay our attention to the rhombic classes, for which we need to check the copositivity of three second-order tensors and a sixth-order tensor. Since the main tasks in checking our conditions is to verify the nonnegativity of the smallest $Z$ eigenvalues of some fourth-order and sixth-order three-dimensional tensors, we describe the direct method for finding the $Z$-eigenvalues of a three-dimensional tensor, as proposed in $[15,16]$.

As we mentioned in the introduction, for certain classes of anisotropic elastic materials several conditions for the strong ellipticity of tensors have been established in the literature, which are "explicit" in the sense that they are inequalities of the elements of the tensor. In our method, we need to check the positivity of the smallest $Z$-eigenvalues of some fourth and sixth order tensors, which have no "explicit" representations in terms of the elements of the original tensor. Thus, our conditions are "implicit" in this sense (even for symmetry classes where there are a few non-zero elements of the tensor). Nevertheless, $Z$-eigenvalues can be obtained easily with the help of a computer. That is, we give a numerical method for computing all $Z$-eigenvalues of an even tensor with dimension 3 . Therefore, the method described here provides an easy way to check the strong ellipticity for general anisotropic elastic materials. From this point of view, our method is complementary to those for certain classes of anisotropic elastic materials in the current literature. 


\section{References}

1. Abeyaratne, R.C.: Discontinuous deformation gradients in plane finite elastostatic of imcompressible materials. J. Elast. 10, 255-293 (1980)

2. Chiriţă, S., Ciarletta, M.: Spatial estimates for the constrained anisotropic elastic cylinder. J. Elast. 85, 189-213 (2006)

3. Chiriţă, S., Danescu, A., Ciarletta, M.: On the strong ellipticity of the anisotropic linearly elastic materials. J. Elast. 87, 1-27 (2007)

4. Cox, D., Little, J., O’Shea, D.: Using Algebraic Geometry. Springer, New York (1998)

5. Dacorogna, B.: Necessary and sufficient conditions for strong ellipticity for isotropic functions in any dimension. Discrete Contin. Dyn. Syst. Ser. B 1, 257-263 (2001)

6. Gurtin, M.E.: The linear theory of elasticity. In: Truesdell, C. (ed.) Handbuch der Physik, vol. VIa/2. Springer, Berlin (1972)

7. Knowles, J.K., Sternberg, E.: On the ellipticity of the equations of non-linear elastostatics for a special material. J. Elast. 5, 341-361 (1975)

8. Merodio, J., Ogden, R.W.: Instabilities and loss of ellipticity in fiber-reinforced compressible nonlinearly elastic solids under plane deformation. Int. J. Solids Struct. 40, 4707-4727 (2003)

9. Ni, G., Qi, L., Wang, F., Wang, Y.: The degree of the E-characteristic polynomial of an even order tensor. J. Math. Anal. Appl. 329, 1218-1229 (2007)

10. Padovani, C.: Strong ellipticity of transversely isotropic elasticity tensors. Meccanica 37, 515-525 (2002)

11. Payton, R.G.: Elastic Wave Propagation in Transversely Isotropic Media. Nijhoff, Dordrecht (1983)

12. Qi, L.: Eigenvalues of a real supersymmetric tensor. J. Symb. Comput. 40, 1302-1324 (2005)

13. Qi, L.: Rank and eigenvalues of a supersymmetric tensor, a multivariate homogeneous polynomial and an algebraic surface defined by them. J. Symb. Comput. 41, 1309-1327 (2006)

14. Qi, L.: Eigenvalues and invariants of tensors. J. Math. Anal. Appl. 325, 1363-1377 (2007)

15. Qi, L., Wang, Y., Wang, F.: A global homogenous polynomial optimization problem over the unit sphere. Working Paper, Department of Applied Mathematics, The Hong Kong Polytechnic University (2007)

16. Qi, L., Wang, F., Wang, Y.: Z-eigenvalue methods for a global polynomial optimization problem. Math. Program. 118, 301-316 (2009)

17. Rosakis, P.: Ellipticity and deformations with discontinuous deformation gradients in finite elastostatics. Arch. Ration. Mech. Anal. 109, 1-37 (1990)

18. Simpson, H.C., Spector, S.J.: On copositive matrices and strong ellipticity for isotropic elastic materials. Arch. Ration. Mech. Anal. 84, 55-68 (1983)

19. Walton, J.R., Wilber, J.P.: Sufficient conditions for strong ellipticity for a class of anisotropic materials. Int. J. Non-Linear Mech. 38, 441-455 (2003)

20. Wang, Y., Aron, M.: A reformulation of the strong ellipticity conditions for unconstrained hyperelastic media. J. Elast. 44, 89-96 (1996)

21. Zee, L., Sternberg, E.: Ordinary and strong ellipticity in the equilibrium theory of impressible hyperelastic solids. Arch. Ration. Mech. Anal. 83, 53-90 (1983) 